\title{
Delayed Detached-Eddy Simulation of the Transonic Flow Around a Supercritical Airfoil in the Buffet Regime
}

\author{
F. Grossi, M. Braza and Y. Hoarau
}

\begin{abstract}
In the present paper a Delayed Detached-Eddy Simulation (DDES) based on the strain-adaptive linear Spalart-Allmaras (SALSA) model is performed in order to investigate the transonic flow over the OAT15A supercritical airfoil within the buffet regime. The results are compared with 2D and 3D-URANS computations using the SALSA model, as well as with experimental data. This study shows improvements achieved in the prediction of the flow unsteadiness and statistics by means of the DDES.
\end{abstract}

\section{Introduction}

In the transonic flow around an airfoil, specific combinations of the Mach number with the angle of attack may engender a large-amplitude periodic motion of the shock-wave known as transonic buffet. The phenomenon occurs from the amplification of a global instability and is self-sustained by a complex interaction between the shock-wave and the boundary layer influenced by the von Kàrmàn instability in the near wake as shown by Bouhadji and Braza [1] and Bourdet et al [2]. In a fluidstructure scenario, this low-frequency aerodynamic instability can rapidly lead to structural vibrations (buffeting). In the case of wings, the phenomenon usually appears on the suction side creating high variations of the aerodynamic loads in flight conditions that can be close to the cruising regime of the aircraft. Therefore, the accurate prediction of the onset of the buffet is of great interest for aeronautical design. A review of the most significant studies concerning the transonic buffet over

F. Grossi - M. Braza

Institut de Mécanique des Fluides de Toulouse - UMR 5502. Toulouse, France.

e-mail: fernando.grossi@imft.fr

Y. Hoarau

Institut de Mécanique des Fluides et des Solides de Strasbourg. Strasbourg, France.

e-mail: hoarau@unistra.fr 
the last decades was given by Lee [7]. In addition to the physical analysis of the instability for circular-arc airfoils first studied by Levy [8] and Marvin et al [9], the author proposes a possible mechanism for the self-sustained shock oscillations over supercritical airfoils. According to Lee, downstream propagating waves created at the foot of the shock reach the trailing edge and generate new upstream moving waves which interact with the shock, providing the energy needed to sustain the buffet mode.

Jacquin et al [5] studied the transonic buffet over a 12.3\%-thick supercritical airfoil, the OAT15A. The experiments were performed in a transonic wind-tunnel at the ONERA-Meudon centre, France. The tunnel had a square test section of $0.78 \times 0.78$ $\mathrm{m}^{2}$ with adaptive upper and lower walls to account for the displacement created by the boundary layers. The model had a chord of $0.23 \mathrm{~m}$ and a thick trailing edge of $5 \times 10^{-3} c$, giving an aspect ratio of 3.4. Transition was fixed on both upper and lower surfaces at a distance $x / c=0.07$ from the leading edge using carborundum strips. The freestream Mach and Reynolds numbers were 0.73 and $3 \times 10^{6}$, respectively, with stagnation conditions of $300 \mathrm{~K}$ and $10^{5} \mathrm{~Pa}$. By means of flow visualization techniques and spectral analysis, the authors showed that the transonic buffet is essentially two-dimensional although some three-dimensional patterns were reported. Moreover, they detected the buffet onset at the angle of attack of $3.1^{\circ}$, with the largescale movement of the shock-wave appearing only for $3.5^{\circ}$. This incidence was then chosen for detailed flow field measurements using Laser Doppler Velocimetry, static pressure orifices and unsteady pressure transducers distributed over the model.

The shock-wave/boundary layer interaction in unsteady flows is a great challenge for turbulence modelling. In the OAT15A case, the prediction of the shock motion at the experimental angle of attack is a particularly arduous task. At this condition, the turbulence model should be sensitive enough to permit the shock-induced separation to extend up to the trailing edge and then reattach the boundary layer in a cyclic way, coupled with the shock-wave oscillation. In previous studies, Thiery and Coustols [14] compared different RANS models and investigated the influence of the test section walls on the flow. Using a two-dimensional approach, they found that the original Spalart-Allmaras [12] (SA) model led to steady state at the experimental angle of attack. However, by accounting for the effects of the upper and lower walls, the same model became able to reproduce the unsteadiness. Using the $k-\omega$ Shear Stress Transport model of Menter [10] (SST), the simulations were successful in predicting the shock motion for both the free air and the confined 2D configurations. By a 3D approach in which the sidewalls were also simulated, the mean and RMS pressure distributions over the airfoil obtained with the SST were in good agreement with the experiments. In all simulations the computed frequencies were close to the experimental value, ranging from 71 to $78 \mathrm{~Hz}$ and decreasing by $3 \mathrm{~Hz}$ when the upper and lower walls were considered. Their study concluded that the modelling of the wind tunnel walls should not be determinant for the evaluation of the capability of a turbulence model in predicting the shock-wave motion in the OAT15A case.

A zonal detached-eddy simulation (zonal-DES) of the OAT15A was performed by S. Deck [3] as one of the few applications of hybrid RANS-LES methods to flows presenting thin-layer separation. The zonal approach has user-defined RANS and 
LES zones and avoids switching to the LES inside the boundary layer, which was explicitly treated in RANS as well as the shock-wave/boundary layer interaction. Deck compared the zonal-DES with 2D-URANS and standard DES computations using the SA model with the transition terms turned off. At the experimental angle of attack, all URANS simulations led to steady flow, requiring an incidence of $4.5^{\circ}$ to trigger the buffet. The DES also produced a steady solution at $3.5^{\circ}$ and needed an angle of $4^{\circ}$ to obtain unsteadiness. Indeed, the zonal-DES was the only method able to capture the shock motion at the experimental conditions, with the periodic oscillations being well reproduced and the spectra of the pressure fluctuations over the airfoil comparing favorably with the measurements.

The objective of the present work is to apply the Delayed Detached-Eddy Simulation (DDES) [13] hybrid method to the transonic buffet flow over the OAT15A at the experimental angle of attack. The DDES is adopted to obtain a richer statistical content than in URANS, concerning the resolved turbulence in respect of finer-scale three-dimensional structures. Moreover, in the buffet case, special attention should be paid to the issue of modeled-stress depletion [13] (MSD) because the boundary layer becomes substantially thick due to the interaction with the shock, especially before separation occurs. The effect of the increased resolved turbulence on the buffet prediction is evaluated by comparing the DDES results with 2D and 3D-URANS simulations in respect to the amplitude of the shock-wave motion, the shock's mean position, local measurements of pressure fluctuations and velocity fields.

\section{Turbulence modelling}

As already mentioned in the previous section, the papers by Deck [3] and Thiery and Coustols [14] on the buffet over the OAT15A airfoil report steady state solutions when using the original SA model at the experimental angle of attack of $3.5^{\circ}$. In order to capture the flow unsteadiness at this particular incidence, the strain-adaptive linear Spalart-Allmaras (SALSA) model of Rung et al [11] has been chosen in the present work. The SALSA is a modified version of the SA model sensitized to nonequilibrium effects, reducing the eddy viscosity in regions where the turbulence production is not equal to the dissipation rate. The SST model and a SST-based DDES approach have been tried as well, leading to steady flow.

In the SALSA model, the transport equation for the modified eddy viscosity is:

$$
\frac{D \tilde{v}}{D t}=\tilde{c}_{b 1} \tilde{S} \tilde{v}+\frac{\partial}{\partial x_{k}}\left[\left(v+\frac{\tilde{v}}{\sigma}\right) \frac{\partial \tilde{v}}{\partial x_{k}}\right]+\frac{c_{b 2}}{\sigma} \frac{\partial \tilde{v}}{\partial x_{k}} \frac{\partial \tilde{v}}{\partial x_{k}}-\tilde{c}_{w 1} f_{w}\left(\frac{\tilde{v}}{d}\right)^{2} .
$$

Besides, the following variables are redefined:

$$
\tilde{S}=S\left(\frac{1}{\chi}+f_{v 1}\right), \quad r=1.6 \tanh \left[0.7\left(\frac{\Psi}{\tilde{S}}\right)\right], \quad \Psi=\sqrt{\frac{\rho_{0}}{\rho}}\left(\frac{\tilde{v}}{\kappa^{2} d^{2}}\right) .
$$


where

$$
S=\sqrt{2 S_{i j} S_{i j}}, \quad S_{i j}=\frac{1}{2}\left[\left(\frac{\partial U_{i}}{\partial x_{j}}+\frac{\partial U_{j}}{\partial x_{i}}\right)\right]-\frac{1}{3} \frac{\partial U_{k}}{\partial x_{k}} \delta_{i j} .
$$

The most important feature of the model is the transformation of the $c_{b 1}$ coefficient, which is no longer constant. Instead, it becomes a function of the strain-rate and of other coefficients as follows:

$$
\begin{gathered}
\tilde{c}_{b 1}=0.1355 \sqrt{\Gamma}, \quad \Gamma=\min [1.25, \max (\gamma, 0.75)], \quad \gamma=\max \left(\alpha_{1}, \alpha_{2}\right), \\
\alpha_{1}=\left[1.01\left(\frac{\tilde{v}}{\kappa^{2} d^{2} S}\right)\right]^{0.65}, \quad \alpha_{2}=\max \left[0,1-\tanh \left(\frac{\chi}{68}\right)\right]^{0.65} .
\end{gathered}
$$

As a consequence, the $c_{w 1}$ coefficient is computed by the function $\tilde{c}_{w 1}=\tilde{c}_{b 1} / \kappa^{2}+$ $\left(1+c_{b 2}\right) / \sigma$. All other terms and constants are identical to the original SA model.

For the SALSA-based DDES approach, the distance to the closest wall $d$ in the SALSA model is replaced by the DDES length scale:

$$
l_{D D E S}=d-f_{d} \max (0, d-0.65 \Delta)
$$

where:

$$
f_{d} \equiv 1-\tanh \left[\left(8 r_{d}\right)^{3}\right], \quad r_{d} \equiv \frac{\tilde{v}}{S \kappa^{2} d^{2}}, \quad S=\sqrt{\frac{\partial u_{i}}{\partial x_{j}} \frac{\partial u_{i}}{\partial x_{j}}}
$$

\section{Numerical Method}

All simulations have been carried out using the NSMB - Navier-Stokes Multiblock solver on SGI Altix ICE 8200 supercomputers. The complete system of the NavierStokes equations has been solved on a multiblock structured grid. The spacial discretisation scheme for the convective fluxes was the third-order AUSM+ [6] and the time discretisation used an implicit dual-time stepping technique, ensuring secondorder time accuracy. A physical time step of $0.5 \mu$ s has been adoped (corresponding to $\approx 5 \times 10^{-4} c / U_{\infty}$ ). The linear system has been solved by the LU-SGS method.

The grid used was constructed based on the $\mathrm{C}-\mathrm{H}$ grid employed by Deck [3]. The farfield was maintained at 80 chords from the airfoil and the number of gridpoints in 2D was increased from 110.000 to about 130.000 in order to favor the LES mode of the DDES (see figures 1(a) and 1(b)). A preliminary computation in steady conditions at an angle of attack of $2.5^{\circ}$ indicated an $y^{+}$always smaller than 0.6 over the entire airfoil. The $3 \mathrm{D}$ grid was then obtained by duplication of the 
2D grid in the spanwise direction. The choice for the span length followed Deck's analogy with a backward facing step [3] of height $H$. In order to capture the larger transverse wavelengths in the flow past the step, a minimum width equivalent to $4 H$ is needed. Assuming that in the airfoil case and at low angles of attack the height of the separated area is about a half of the airfoil width $e$, a span $b$ of one quarter of chord would be sufficient for the OAT15A, since $b \approx 2 e$ and $e=0.123 c$. Hence, 64 equally-spaced cells were distributed over a span of $0.26 c$ in order to keep $\Delta x \approx \Delta z$ $(\approx 0.4 c / 100)$, yielding a final grid with approximately 8.3 million control volumes (see figure 1(c)). Due to the small span adopted, periodic conditions were used at the lateral boundaries.

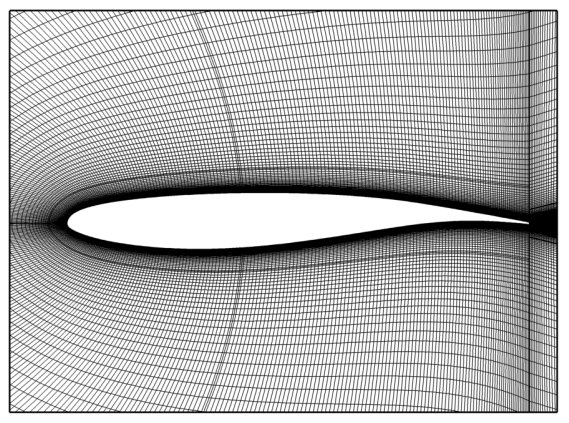

(a) 2D grid with 110.000 points

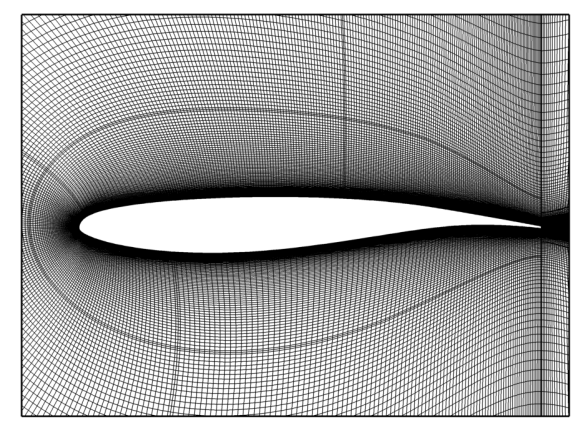

(b) $2 \mathrm{D}$ grid with 130.000 points

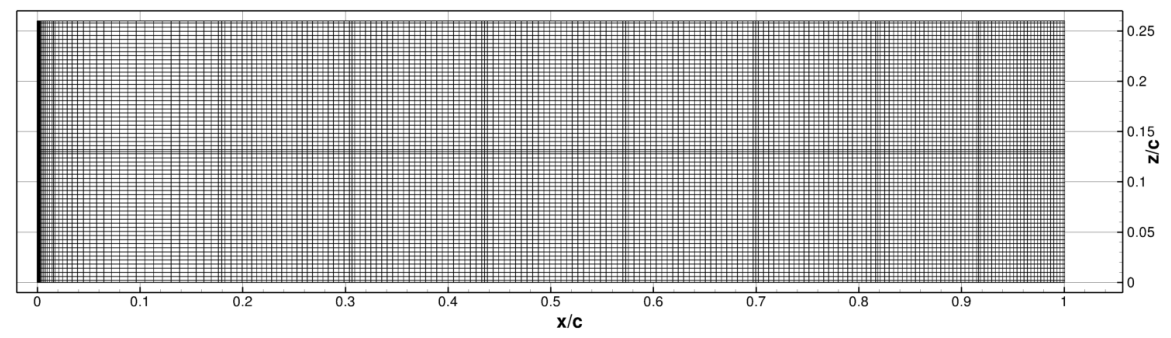

(c) 3D grid over the upper surface

Fig. 1 Detail of the grid around the OAT15A airfoil

An important issue is how the RANS and LES behave in the DDES for the present grid. Considering first a standard DES for the sake of comparison, in which the RANS length scale is defined as the distance from the closest wall (as in the case of the DES97[13]), the boundary between the RANS and LES regions remains static and is a function of the grid exclusively. For the present grid, the RANS region would be a very thin layer around the airfoil, especially near the trailing edge where the grid-space is particularly fine (see figure 2(a)). Since in the transonic buffet the boundary layer becomes much thicker than this layer, the LES would be activated in zones where there is no sufficient grid resolution for a LES. By using the DDES, 
the RANS boundary becomes dynamic and much thicker than in DES, as illustrated in figure 2(b) for an instantaneous field. The present formulation forces most of the boundary layer and of its interaction with the shock-wave to be treated in RANS.

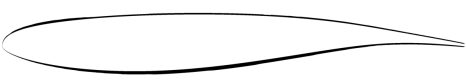

(a) Standard DES

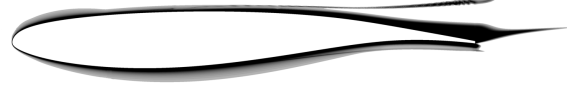

(b) Delayed DES

Fig. 2 Regions of RANS (in black) and LES (in white) modes around the OAT15A profile

\section{Results and Discussion}

By using the SALSA version of the SA model, the transonic buffet over the OAT15A was captured at the experimental angle of attack of $3.5^{\circ}$ by URANS (2D and 3D) as well as by DDES. In all cases, the global dynamics of the phenomenon was well reproduced, with the coupling between the shock-wave/boundary layer interaction and the separation/reattachment process of the boundary layer agreeing well with the description given in reference [5].

Figure 3 illustrates some instantaneous iso-surfaces of the Q-criterion $(Q=$ $\left.\frac{1}{2}\left(\left\|\Omega^{2}\right\|-\left\|S^{2}\right\|\right)\right)$ for $Q\left(c / U_{\infty}\right)^{2}=10$ obtained with the DDES. Figure 3(a) corresponds to the moment of maximum lift, with the boundary layer attached over the upper surface and the shock located about its most downstream position. Hence, a progressive growth of the boundary layer takes place together with the shock-wave motion towards the leading edge. During the movement, the boundary layer separates from the shock foot extending downstream to the trailing edge. This engenders quasi-two-dimensional structures (3(b)) which become three-dimensional due to the amplification of the secondary instability (figure 3(c)). In this separated period of the buffet, the DDES gives a much richer content of smaller-scale turbulent structures than URANS. After the shock reaches its most upstream position and starts moving downstream, the boundary layer reattaches and the vortex shedding ceases (3(d)).

The time history of the lift coefficient indicates quasi-sinusoidal patterns of constant period and is presented in figure 4(a) for the three approaches. In the URANS simulations, the predicted lift fluctuations reach $\approx 22 \%$ of the mean lift, with the computed buffet frequencies approximately equal to $69 \mathrm{~Hz}$. This value is in very good agreement with the experimental frequency reported in reference [5] $(\approx 70$ $\mathrm{Hz}$ ). The DDES exhibits an increase of the mean lift and a reduction on the level of the fluctuations to about $16 \%$, and a higher frequency of $\approx 78 \mathrm{~Hz}$. This behaviour is closely related to a mean shock-wave position located slightly downstream and to a smaller shock motion range than in URANS. 


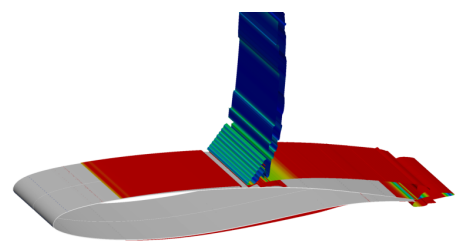

(a)

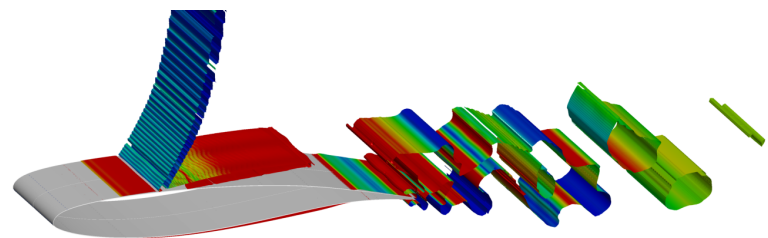

(b)

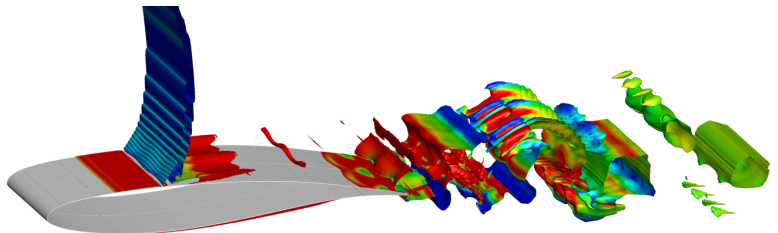

(c)

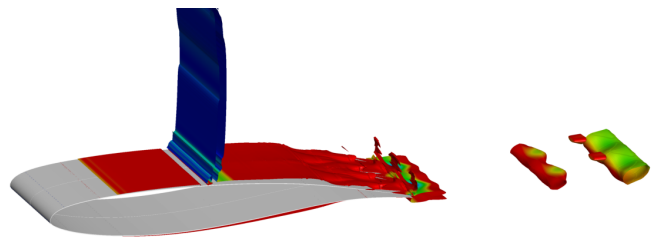

(d)

Fig. 3 Iso-surfaces of the Q-criterion for $Q\left(c / U_{\infty}\right)^{2}=10$ colored by the vorticity magnitude.

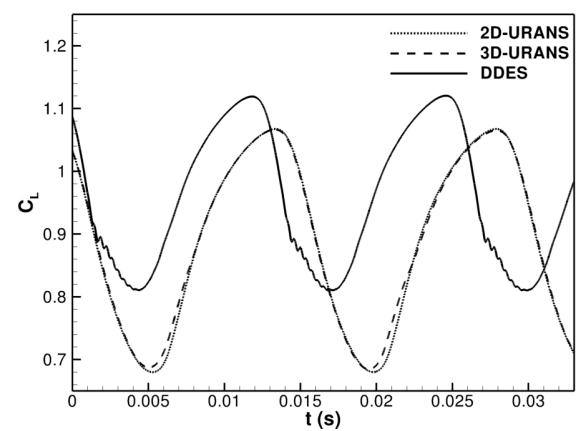

(a)

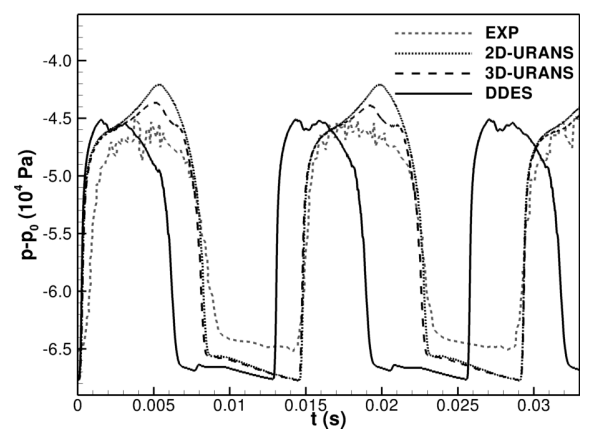

(b)

Fig. 4 Time evolution of the lift (a) and of the pressure at $x / c=0.45$ on the upper surface (b) 
The analysis of local properties of the flow gives further information about the performance of the DDES method on the buffet prediction. Figure 4(b) shows how the pressure oscillates on a point located at $x / c=0.45$ over the upper surface of the airfoil. The flattened low-pressure zones correspond to phases in which the shock is located downstream of the monitor point. During this period of the flow, the value estimated by the numerical simulations is lower than in the experiments. The sudden increase in pressure indicates the moment when the shock-wave passes by $x / c=0.45$ and places the point inside the compression zone. It is interesting to notice the progressive decrease of the maximum pressure from 2D to 3D-URANS, and to DDES. The latter compares quite well with the experiments and is able to capture the secondary oscillations caused by upstream moving waves generated at the trailing edge due to the impact of unsteady structures during the separated period of the flow. The DDES method can reproduce those higher frequency effects in much more detail because of the improved resolution of smaller scales. These secondary fluctuations, for instance, influence even the airfoil global lift and can be seen in the lift curve in figure 4(a) near the phases of minimum lift (maximal flow separation).

Figure 5(a) illustrates the profiles of the mean pressure coefficient over the upper and lower surfaces of the airfoil. The inclined aspect of the shock zone is due to the averaging procedure and roughly indicates the range of the shock motion, which is too wide in all cases. All approaches easily reproduce the experimental pressure on the lower surface and slightly overestimate the pressure of the supersonic plateau. For the DDES, the pressure predicted around the trailing edge is a bit lower than the URANS results.

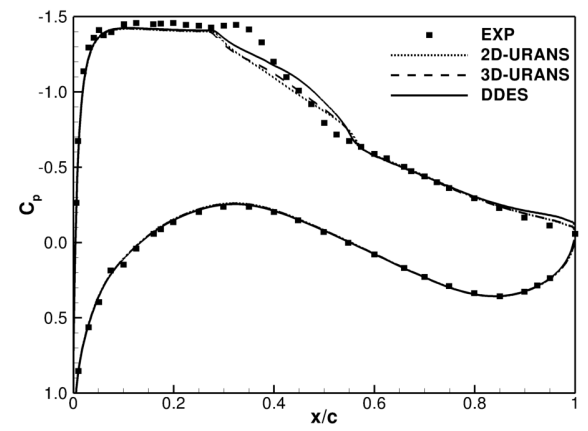

(a)

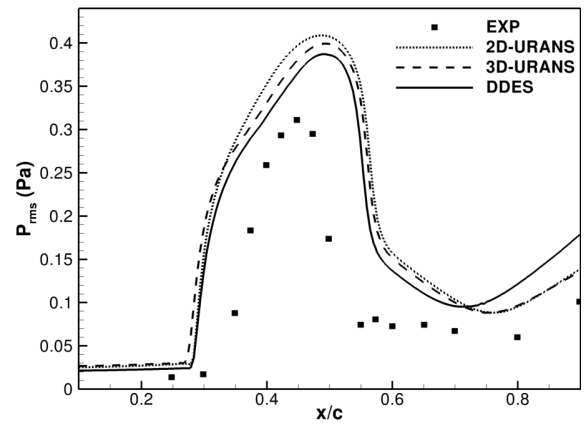

(b)

Fig. 5 Mean pressure coefficient over the airfoil (a) and RMS pressure on the upper surface (b)

The distribution of the RMS pressure over the suction side of the OAT15A is given in figure 5(b). In the region of high pressure fluctuation, the DDES gets closer to the experiments than URANS and predicts a narrower shock-wave motion range, although the magnitudes remain excessive. This conclusion is also supported by the flowfield visualization in figure 6, which illustrates the RMS fields of the longitu- 
dinal velocity $u$ for the 2D-URANS and the DDES. Compared to URANS, the velocity fluctuations for DDES are lower and stay confined within a slenderer region. The DDES gets closer to the experimental curve also in the first part upstream the shock. However, over the rear portion of the airfoil, the hybrid method particularly overpredicts the RMS levels, specially near the trailing edge. This indicates intense fluctuations caused by the unsteadiness of an increased separated region which can also be noticed by means of the velocity fields in figure 6 , and is the reason for the lower trailing edge pressure shown in figure 5(a) for the DDES. Comparing only the two URANS computations, the results are similar over the entire airfoil as for the mean pressure coefficient. Indeed, the 3D-URANS exhibits already a small decrease of the pic level compared to 2D, suggesting a transfer of some amount of energy towards three-dimensional structures.

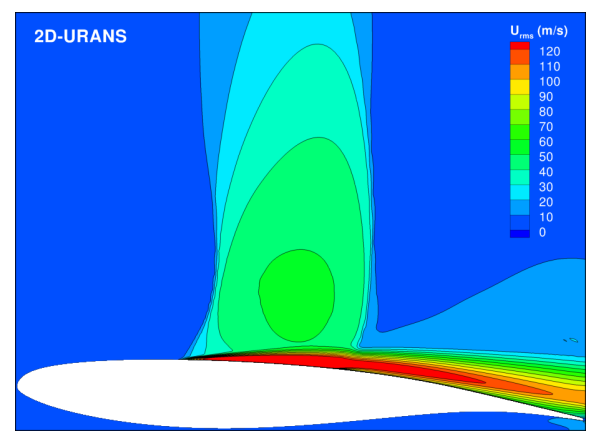

(a)

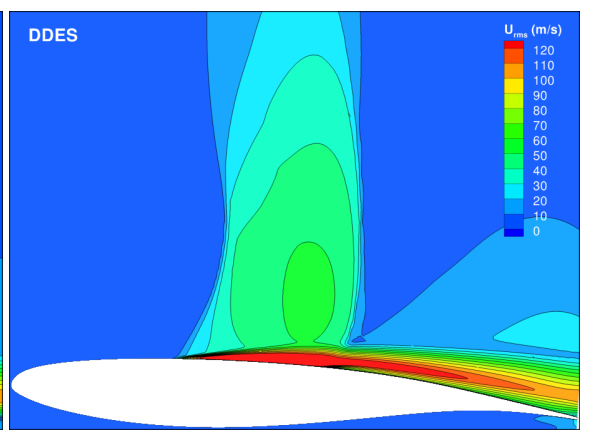

(b)

Fig. 6 RMS fields of the longitudinal velocity $u$ for 2D-URANS (a) and DDES (b)

The analysis of the results indicates that by accounting for the effect of smaller turbulence scales by means of the DDES an overall improvement on the prediction of the transonic buffet over the OAT15A is reached. This is true for the amplitudes of the shock-wave motion and particularly for the fluctuating properties of the flow. Indeed, the extrema of the shock motion range remains primarily related to the capability of the RANS model in predicting separation. The mean position of the shock during buffet also mostly depends on the RANS model.

\section{Summary}

The transonic buffet over the OAT15A supercritical airfoil has been investigated by a Delayed Detached-Eddy Simulation based on the strain-adaptive linear SpalartAllmaras model. The results have been compared with 2D and 3D-URANS computations using the same model as well as to experimental data. The flow unsteadiness has been captured at the experimental angle of attack and the global dynamics of 
the buffet phenomenon has been well predicted by all approaches. The computed frequencies are close to the experimental value. The shock-wave motion is overestimated in all cases; improved prediction is achieved through DDES. With the effect of smaller turbulence scales resolved by DDES, the fluctuating properties of the flow and the vortex structures in the wake are better predicted compared to URANS. For the latter, only small differences are noticed between 2D and 3D.

Acknowledgements The present work has been performed in the context of the European research program ATAAC - Advanced Turbulence Simulation for Aerodynamic Application Challenges coordinated by the DLR, Germany. The authors are grateful to the national computing centres of France, CINES, CALMIP and IDRIS, for the computing time allocation.

\section{References}

1. Bouhadji, A., Braza, M.: Organised modes and shock-vortex interaction in unsteady viscous transonic flows around an aerofoil Part I: Mach number effect. Computers \& Fluids 32, 12331260 (2003)

2. Bourdet, S., Bouhadji, A., Braza, M., Thiele, F.: Direct Numerical Simulation of the ThreeDimensional Transition to Turbulence in the Transonic Flow around a Wing. Flow, Turbulence and Combustion 71, 203-220 (2003)

3. Deck, S.: Numerical Simulation of Transonic Buffet over a Supercritical Airfoil. AIAA Journal 43(7), 1556-1566 (2005)

4. Edwards, J.R., Chandra, S.: Comparison of Eddy Viscosity-Transport Turbulence Models for Three-Dimensional, Shock-Separated Flowfields. AIAA Journal 34(4), 756-763 (1996)

5. Jacquin, L., Molton, P., Deck, S., Maury, B., Soulevant, D.: Experimental Study of Shock Oscillation over a Transonic Supercritical Profile. AIAA Journal 47(9), 1985-1994 (2009)

6. Liou, M-.S.: A Sequel to AUSM: AUMS+. Journal of Computational Physics 129, 364-382 (1996)

7. Lee, B.H.K.: Self-sustained shock oscillations on airfoils at transonic speeds. Progress in Aerospace Sciences 37, 147-196 (2001)

8. Levy Jr, Lionel L.: Experimental and Computational Steady and Unsteady Transonic Flows about a Thick Airfoil. AIAA Journal 16(6), 564-572 (1978)

9. Marvin, J. G., Levy Jr, L. L., Seegmiller, H. L.: Turbulence Modeling for Unsteady Transonic Flows. AIAA Journal 18(5), 489-496 (1980)

10. Menter, F.R.: Two-Equation Eddy-Viscosity Turbulence Models for Engineering Applications. AIAA Journal 32(8), 1598-1605 (1994)

11. Rung, T., Bunge, U., Schatz, M., Thiele, F.: Restatement of the Spalart-Allmaras EddyViscosity Model in Strain-Adaptive Formulation. AIAA Journal 41(7), 1396-1399 (2003)

12. Spalart, P.R., Allmaras, S.R.: A one-equation turbulence model for aerodynamic flows. La Recherche Aérospatiale 1, 5-21 (1994)

13. Spalart, P.R., Deck, S., Shur, M.L., Squires, K.D., Strelets, M.Kh., Travin, A.: A new version of detached-eddy simulation, resistant to ambiguous grid densities. Theoretical and Computational Fluid Dynamics 20, 181-195 (2006)

14. Thiery, M., Coustols, E.: Numerical prediction of shock induced oscillations over a 2D airfoil: Influence of turbulence modelling and test section walls. International Journal of Heat and Fluid Flow 27, 661-670 (2006)

15. van Leer, B.: Towards the Ultimate Conservative Difference Scheme, V. A Second Order Sequel to Godunov's Method. Journal of Computational Physics 32, 101136 (1979) 